\title{
A Canadian Urological Association best practice statement on chronic scrotal pain
}

Keith A. Jarvi, MD, FRCSC ${ }^{1}$; Christopher Wu, MD, FRCSC ${ }^{1}$; J. Curtis Nickel, MD, FRCSC ${ }^{2}$; Trustin Domes, MD, Med, FRCSC ${ }^{3}$; John Grantmyre, MD, FRCSC ${ }^{4}$; Armand Zini, MD, FRCSC $^{5}$

${ }^{1}$ Sinai Health System, University of Toronto, Toronto, ON; ${ }^{2}$ Queen’s University, Kingston, ON; ${ }^{3}$ University of Saskatchewan, Saskatoon, SK; ${ }^{4}$ Dalhousie University, Halifax, NS; ${ }^{5}$ McGill University, Montreal, QC; Canada

Acknowledgements: The authors would like to thank Susan Lau for her efforts in organizing the collaboration between authors, editing the article, and preparation for submission.

Cite as: Can Urol Assoc J 2018 Feb. 23; Epub ahead of print. http://dx.doi.org/10.5489/cuaj.5238

Published online February 23, 2018

$* * *$

\section{Introduction}

Chronic scrotal pain (CSP) is poorly understood, with relatively limited information published on the incidence, natural history or therapies for this chronic condition. Estimates of the frequency of CSP range from 0.4- 4.75\%, but these estimates are based on studies of CSP within specific groups of men and may not reflect the true incidence of CSP among the general population of men ${ }^{1-3}$. Unfortunately, this chronic condition is frequently extremely frustrating for both patients and clinicians, with patients often seeing multiple clinicians to seek treatment, with one study finding that the average CSP patient will have seen a mean of 4.5 urologists for the condition and undergo an average of 7.2 diagnostic investigations ${ }^{4}$.

Described as "chronic orchialgia" 5 or testicular pain syndrome ${ }^{6}$, it is now recognized that scrotal pain may arise not only from the testicles, but can involve adjacent paratesticular structures such as the epididymis and vas deferens or pain may be referred from conditions involving the spermatic cord or the retroperitoneum. Therefore, the broader term chronic scrotal pain is more descriptive when referring to this condition ${ }^{1,7}$.

Similar to other chronic pain conditions, CSP has been shown to negatively impact the affected individual's quality of life. More than 50\% of patients with CSP report limitations to their daily activities, limited ability to work, and decreased sexual activity ${ }^{8}$. Depression is also frequently associated with CSP, with $40 \%$ of patients with CSP reporting depressive symptoms more than half of the time ${ }^{8}$. In a study of 48 men diagnosed with CSP and no organic findings, Schover et al. found that many of these patients were diagnosed with psychological disorders, including somatization disorder in 50\% and depression in $27 \%{ }^{9}$. Up to a third of these patients were socially isolated, and 18\% had an emotional event occurrence around the onset of pain.

The concept of chronic pain has now shifted from a biomedical model to a biopsychosocial one. Studies have shown that the level of social support, expectation of pain, 
interference with work or other activities, and past memories all affect an individual's experience of pain ${ }^{10}$. CSP is often debilitating and is associated with depression, anxiety, sexual dysfunction, and overall decreased quality of life $\mathrm{e}^{11}$.

Currently, there is a lack of widely accepted published guidelines for the evaluation and management of men presenting with CSP. Such guidelines will provide a framework to help support clinicians struggling to manage men with this challenging condition. The purpose of this document is to thoroughly review the current literature on CSP and provide a CUA best practice statement to help direct the evaluation and management of men presenting with this oftendebilitating condition.

\section{Methodology}

A comprehensive review of reports and studies on CSP was performed using PubMed, EMBASE, MEDLINE and Cochrane library databases from January 1990 - June 2017. The bibliographies of relevant articles were also carefully reviewed and searched for further significant articles. Consensus statements and guidelines from the European Association of Urology (EAU), National Institute for Health and Care Excellence (NICE), Canadian Pain Society (CPS), and the American Academy of Neurology (AAN) were incorporated. Articles were reviewed using the Grading of Recommendations Assessment, Development, and Evaluation (GRADE) approach for summarizing the evidence and making recommendations.

\section{Definition}

Chronic scrotal pain is defined as intermittent or constant, unilateral or bilateral pain localized to the scrotal structures, 3 months or longer in duration that significantly interferes with daily activities of the patient and prompts him to seek medical attention ${ }^{7,12-14}$.

\section{Epidemiology}

Despite how common the condition seemingly presents in urology practice, there are few studies reporting on the frequency of CSP in the general population. Ciftci et al. reviewed a total of 2375 men seen in urology outpatient clinics in Turkey who were not being seen for CSP ${ }^{15}$. They used questionnaires to determine if the men had CSP. They reported that CSP was identified in $4.75 \%$ of patients, presenting with a mean age of $32.5 \pm 10$ years. A Canadian study reported that $4.3 \%$ of the men presenting to a male infertility clinic in Toronto reported having $\operatorname{CSP}^{1}$. A Swiss study reported an estimated incidence of CSP of 4/1000, though CSP accounted for $2.5 \%$ of all visits to Swiss urologists ${ }^{2}$. However, this was based on a questionnaire survey sent out to solicit urologist's recollections of the numbers of men with CSP that they treated. None of the above provides a general population based estimate of the frequency of CSP. A general population study from Israel looking at young men in military service, reported that $0.8 \%$ of patients accessed care for the treatment of $\operatorname{CSP}^{15}$. This is not a general estimate of the frequency of CSP in men of all ages and only captures men who accessed care for the management of CSP, not the frequency of the CSP in the population (many men with CSP do not seek medical care for the condition). 
How many men with CSP are assessed by urologists? The Canadian PIE study examined the number of men presenting to urology practices in Canada with the diagnosis of prostatitis interstitial cystitis and epididymitis. In this study, a randomly selected list of urologists representing both community and academic practice, audited a 2-consecutive week period of outpatient visits. A diagnosis of epididymitis was identified in $0.9 \%$ of men seeking urology consultations, with $80.7 \%$ classified as chronic ${ }^{16}$.

\section{Etiology}

The pathophysiology of CSP is still poorly understood. Pain to the scrotal structures may be either direct or referred by other structures in the same segmental nerve distribution.

Pain in general is described as either nociceptive, neuropathic, or they can coexist. Nociceptive pain arises from tissue injury or inflammation, while neuropathic pain is defined as "pain arising as a direct consequence of a lesion or disease affecting the somatosensory system", i.e. arising from the nervous system ${ }^{17}$.

The sensory innervation of the scrotum and scrotal contents occurs via the somatic nerves in the genital branch of the genitofemoral nerve (L1-L2) as well as the ilioinguinal nerve (L1) ${ }^{13}$. The autonomic innervation of the testis is from the presacral ganglia of T10-12, meanwhile those from the epididymis and vas deferens are distributed to T10-L1 segments ${ }^{18}$.

Acute scrotal pain is usually nociceptive and involves the activation of nociceptors within nerve endings by noxious stimuli (such as tissue injury or inflammation). A pain signal is then relayed through myelinated A delta fibers as well as unmyelinated $C$ fibers to reach the dorsal horn of the spinal cord and travel to the brain through the spinothalamic tracts ${ }^{13}$.

What is poorly understood is how chronic pain arises from acute pain. Pain that persists long after the inciting event has healed is a complex process. Certainly, there may be a continued nociceptive input from processes such as ongoing tissue inflammation, infection, or nerve entrapment, however there may also be a pathologic activation of the central nervous system in a process known as central sensitization ${ }^{19}$. With central sensitization, the responsiveness of the neurons increase, leading to activation of pain transmission by normally innocuous inputs (allodynia) or heightened responses to painful stimuli (hyperalgesia).

Several studies have shown that the most common identifiable causes of direct pain to the scrotal structures are a previous vasectomy (found in close to $21 \%$ of patients presenting to a CSP clinic), followed by varicoceles, inguinal hernia repairs, spermatoceles, varicoceles, hydroceles, infections of the epididymis/testis, tumours (testicular or paratesticular) or testicular torsion $^{15,20,21}$. In another study focusing on younger males in military service, the most common identifiable factor was a varicocele, which was detected in over $50 \%$ of men presenting with scrotal pain lasting more than two weeks ${ }^{15}$. Additionally, up to 58\% of men with chronic prostatitis/chronic pelvic pain syndrome (CP/CPPS) also have coexisting scrotal pain ${ }^{22}$. Unfortunately, up to 50\% of patients presenting with CSP will not have an identifiable etiology, making medical or surgical management difficult ${ }^{1,12,23}$.

Common causes of referred pain to the scrotal contents include mid ureteral stones and radiculitis from degeneration of the thoracic and lumbar spine ${ }^{20}$. It is also important to identify 
structures outside of the scrotum (tendons, muscles, ligaments, hernias) which may bring the patient for an assessment of his "CSP", when in fact the pain is due to a non-scrotal source in close proximity to the groin ${ }^{1}$. Other causes of referred scrotal pain include nerve entrapment by either an indirect inguinal hernia, scarring from prior inguinal surgery, or tendonitis of the insertion of the inguinal ligament into the pubic tubercle ${ }^{1,18}$. These should be identified and treatment should be targeted to the referring source.

"Self-palpation" orchitis has also been described, which occurs when a patient anxiously self-examines their testicles and scrotum, with the frequent palpation leading to local testicular pain despite a lack of prior symptoms and an absence of intrascrotal disease ${ }^{24}$.

The relationship between pain and depression is complex, and when questioned about depression, patients may blame everything on their pain ${ }^{25}$. Patients with chronic pain have been observed to have a higher incidence of depressive symptoms, with one Canadian study reporting that over $31 \%$ of men have depressive symptoms more than half the days of the month ${ }^{8}$. Psychogenic pain (or pain without an identifiable cause), has also been reported in a variety of psychological conditions, including depression, anxiety, and schizophrenia ${ }^{9,11}$. However, it is not clear whether the pain symptoms bring about psychiatric distress or whether underlying psychiatric conditions potentiate pain. Catastrophizing may also occur in chronic pain patients, where they experience a set of negative pain-related thoughts when undergoing or anticipating pain $^{26}$. Catastrophizing has been correlated with increased depressive symptoms and increased pain $^{11}$. In one study, patients who catastrophized were found to have negative relationships with social supports and healthcare providers, leading to more helplessness and creating additional barriers in the management of their chronic pain ${ }^{11}$.

\section{Natural history}

As the etiology of CSP can vary significantly, and oftentimes there is no discernible cause for pain, the natural history of the condition remains poorly studied. Many of the patients presenting for management of CSP will have seen other healthcare professionals and tried many empiric treatments without benefit, and the ones that have had successful resolution of pain without intervention are not commonly captured by existing studies.

Summary and recommendations: CSP is defined as intermittent or constant, unilateral or bilateral pain localized to the scrotal structures, 3 months or longer in duration that significantly interferes with daily activities of the patient and prompts him to seek medical attention. Though epidemiologic data is limited, the overall prevalence of CSP in the Canadian population is estimated at $1-4 \%$. Up to $50 \%$ of patients will not have an identifiable etiology for their pain.

\section{Diagnostic evaluation}

History (mandatory, Grade 3C)

A thorough patient history should identify medically important and potentially reversible causes for scrotal pain. The pain should be fully characterized, noting the onset, location, quality, severity, referral and psychosocial impact of the pain. Activities that both aggravate and alleviate 
the pain should be noted, including details on urinary function, sexual function, as well as bowel function. A past medical and surgical history should be elicited from the patient, including any previous scrotal, inguinal, abdominal, or pelvic surgeries. It is also important to ask about prior evaluation and interventions for the presenting pain, as well as whether any prior treatment has brought relief. Any history of psychological, physical, or sexual abuse should be elicited. Aside from identifying potential risk factors for infectious causes of scrotal pain such as epididymoorchitis, men with a history of abuse are at an increased risk of CP/CPPS ${ }^{27}$ (Level of evidence 3, Grade C).

Physical examination (mandatory, Grade 4C)

The physical examination in a patient with CSP should focus not only on the genitalia. The patient should be examined in both standing and supine position, and the examination should begin on the non-painful or less painful side. The scrotal structures should be carefully palpated, focusing on the testes, epididymides, and vasa for any anatomic abnormalities and to localize the source of the scrotal pain. The inguinal area should also be carefully inspected for surgical scars, hernias or areas of tenderness. Care should also be taken to identify tenderness in the region of the adductor insertion which is often found in men presenting for investigation of CSP. A digital rectal examination is essential to assess for any abnormalities of the prostate as well as hypertonicity or point tenderness of the pelvic floor structures ${ }^{7}$ (Level of evidence 4, Grade C). A screening neurological examination of the lower limbs and genitals is often required to assess for sensory deficits and radicular syndromes.

Infection screen (optional, select patients, Grade 3C)

Initial laboratory investigation should be symptom directed. A urinalysis is an optional screening test utilized in multiple series as an initial investigation, though its utility is debated. Costabile analyzed a series of 48 men with orchialgia, of which 33 had urinalysis as part of the initial set of investigations ${ }^{25}$. All the urinalyses in this series were negative and did not change management. However, in a series of men with CSP localized to the epididymis, Nickel et al. found that up to $18 \%$ of men were found to have a urinary tract infection (UTI) ${ }^{28}$. A urinalysis should be ordered in the setting of lower urinary tract symptoms or hematuria (Level of evidence 3, Grade C).

If the patient is at a high risk for sexually transmitted infections (STI) or has complaints of urethral discharge, urethral symptoms, or penile pain then a urethral swab or urine for nucleic acid amplification for Neisseria gonorrhea and Chlamydia trachomatis $(\mathbf{G}+\mathbf{C})$ should be ordered. ${ }^{28,29}$ Other more fastidious organisms such as Mycoplasma and Ureaplasma should also be considered if an infectious source is suspected (Level of evidence 3, Grade C).

In patients with concomitant perineal discomfort or pain suggestive of prostatitis, consideration of standard infection screening studies for $\mathrm{CPPS}^{30}$ can be considered (Level of evidence 3, Grade C).

Questionnaire (optional, Grade 4C) 
The use of validated questionnaires is invaluable for the assessment of symptoms, allowing for the measurement of a baseline as well as evaluation of treatment success. The Chronic Epididymitis Symptom Index is a published questionnaire by Nickel et al. measuring the severity, frequency and impact of chronic epididymitis and can serve as a useful tool for baseline evaluation as well as follow-up of patients ${ }^{28}$ (Level of evidence 3, Grade C). Evaluated and employed in the chronic epididymitis population, the symptom index is separated into two domains, one examining pain frequency and severity, the other drawing from the impact/quality of life questions from the National Institutes of health Chronic Prostatitis Symptom Index (NIHCPSI) ${ }^{29}$ Though not validated specifically for the CSP population, this questionnaire can be easily adapted for use in the broader CSP population.

Scrotal ultrasound (optional, select patients, Grade 3C)

Scrotal ultrasound (US) should be performed if there is a palpable abnormality (such as a mass) in the scrotum or where pain or patient body habitus precludes a proper physical examination $^{31,32}$ (Level of evidence 3, Grade C). Additionally, many patients and clinicians feel more reassured that there is no contributing pathology when a scrotal US is obtained. However, routine scrotal US to evaluate all men with CSP has been subject to debate, given the low yield and detection of unrelated findings in the setting of a normal scrotal examination. Numerous studies have shown that an US for the investigation of scrotal pain (in the setting of a completely normal examination) added no clinically valuable information ${ }^{31-33}$. Hoon Cho et al. performed routine scrotal US in patients who underwent vasectomy with and without post-vasectomy pain and noted no differentiating features in the ultrasound findings between the two patient populations $^{34}$. In another study, Van Haarst et al. reviewed the scrotal US results of 102 patients with CSP (with a normal scrotal examination) and diagnosed 12 subclinical epididymal cysts (less an $0.5 \mathrm{~cm}$ in diameter) and 3 subclinical varicoceles, information which was felt to be of no clinical value to the patients ${ }^{32}$ (Level of evidence 3, Grade C). Although scrotal US is a safe and relatively inexpensive investigation often ordered in the CPS population, studies show that its clinical utility is limited in the setting of a normal scrotal examination and it may detect clinically insignificant findings potentially leading to further investigations or unnecessary procedures $^{32}$. 
Test cord block (optional, Grade 3C)

Spermatic cord blockade can serve as both a diagnostic and a therapeutic measure for patients with idiopathic CSP. This allows the clinician to better determine whether or not the pain is originating from within the scrotum. Most series describe injecting 10-20mL of local anesthetic without epinephrine (lidocaine 1-2\% and/or Marcaine 0.25-0.5\% without epinephrine) just lateral to the pubic tubercle ${ }^{35,36}$. The spermatic cord is held in the upper scrotum taking care to encircle all of the structures of the cord with the urologist's fingers. The vas deferens is usually easily palpated and is typically the most posterior structure in the cord. The local anaesthetic is then injected into and around the spermatic cord as it courses over the symphysis pubis to complete the spermatic cord blockade. Pain relief, if it occurs, usually occurs within minutes and may last hours or even days. The response to the cord block will help differentiate local scrotal pain from referred pain. Several studies have shown that a positive response to a temporary cord block is a useful predictor of sustained pain improvement with microsurgical denervation of the spermatic cord (MDSC) ${ }^{35,37}$ (Level of evidence 3, Grade C).

Psychological evaluation (optional, select patients, Grade 4C)

It is well established that chronic pain significantly affects one's quality of life. The symptoms of depression may present in more than $50 \%$ of patients with CSP, significantly impacting psychosocial functioning ${ }^{1,8}$. Somatization disorders are also prevalent and may be found in up to $50 \%$ of patients ${ }^{9}$. Many patients are also socially isolated and up to $30 \%$ of patients may develop chemical dependency disorders, many self-medicating for their pain ${ }^{9}$.

Referral to a mental health specialist is indicated if: 1) patient endorses significant psychiatric response to ongoing pain, 2) pain affects non-medical aspects of life (relationships, employment, legal issues), or 3) if pain is accompanied by anxiety, depression or significant mental distress ${ }^{11}$ (Level of evidence 4, Grade C.

Testicular function screening (optional, select patients, Grade 4C)

In young patients presenting with CSP and non-proven fertility, a semen analysis may be considered, especially if an infectious etiology is suspected such as a history of STI or epididymo-orchitis. In patients with acute unilateral epididymitis, transient deterioration of semen quality is seen in most patients and permanent damage in a smaller subset of patients. Up to $30-40 \%$ of patients may have ongoing oligozoospermia or azoospermia after an acute episode of epididymo-orchitis lasting up to 2 years ${ }^{38}$. Bacterial infection of the epididymis is associated with fibrosis and obstruction, leading to lasting epididymal damage ${ }^{39}$ (Level of evidence 4, Grade C).

Though there are no studies demonstrating a higher risk of androgen deficiency in men with CSP, serum testosterone testing should be considered if the patient has symptoms of testosterone deficiency or if surgery is contemplated to manage a man's CSP in the future ${ }^{40}$.

Summary and recommendations: Patients with CSP require thorough assessment. Taking a history should be one of the first steps in the assessment of CSP, followed by clinical examination (Expert opinion). As CSP may occur in the setting of a symptomatic 
UTI, STI, or prostatitis, an infection screen should be ordered in the setting of any suspicion of infection (Level of evidence 3, Grade C). Scrotal ultrasound is indicated in the setting of an abnormal physical examination or if pain precludes examination (Level of evidence 3, Grade C). Scrotal ultrasound is optional in the setting of a normal physical examination (Level of evidence 4, Grade D). A test spermatic cord block may differentiate referred versus direct scrotal pain and predict future intervention success, thus is optional (Level of evidence 3, Grade C). Psychiatric referral is recommended in settings where the patient endorses significant psychiatric response to ongoing pain, 2) pain affects nonmedical aspects of life (relationships, employment, legal issues), or 3 ) if pain is accompanied by anxiety, depression or significant mental distress (Expert opinion). Semen analysis is optional in young men with unproven fertility and suspected infectious etiology (Level of evidence 4, Grade C).

\section{Treatment}

Managing men with CSP typically involves a step-wise approach, starting with the least invasive options first and moving to more invasive therapies as needed. Some of the suggested therapies fall outside of the normal practice of urology and referrals to other medical disciplines may be required (such as pain specialists, neurologists, psychiatrists and sport's medicine specialists).

\section{Conservative management (Grade 4C)}

There is currently no level 1 evidence for any treatments available for the management of CSP. As such, little data on the natural history of CSP is available. It is reasonable to attempt a trial of watchful waiting if symptoms are mild. Reassurance that pain does not necessarily mean a serious pathology such as cancer may be able to comfort some patients. Other reasonable conservative treatments include scrotal support, heat or cold therapy, and avoidance of aggravating activities (Level of evidence 4, Grade C).

Physiotherapy (Grade 4C)

Extrapolating from CP/CPPS studies, if concomitant pelvic floor tenderness or dysfunction is present on clinical examination, pelvic floor physiotherapies in the form of perineal/pelvic floor massage or myofascial trigger point release may be suggested as a treatment modality for patients, however further studies are needed before any evidence based-recommendations can be made $^{41}$. Up to $10 \%$ of CSP patients may present with musculoskeletal pain localized to locations such as the conjoint tendon and the adductor muscles, and physiotherapy directed to these areas should be considered ${ }^{1}$ (Level of evidence 4, Grade C).

Furthermore, a frequently overlooked cause of referred testicular pain is through the irritation of the T10-L1 sensory nerve roots. If the patient has concomitant back and scrotal pain, passive and active mobilization of this region of the spine and exercises to improve stretching and strength have demonstrated benefit in case reports ${ }^{42}$ (Level of evidence 4, Grade C). 
Extrapolating from reports on men with CPPS, acupuncture may also represent a safe and potentially efficacious therapy for CSP. In one pilot study, patients with CPPS underwent 2 acupuncture sessions weekly for a total of 8 weeks ${ }^{43}$. A significant decrease in NIH-CPSI scores were found in more than half of the patients. Further study is required to determine the translatability of these results to the specific CSP population (Level of evidence 4, Grade D).

Psychological counseling and therapies (Grade 4C)

Patients with a history of CP/CPPS often present with concomitant CSP. The European Association of Urology placed CSP under the umbrella of CP/CPPS, though the latter is much more widely studied. Research in the treatment of CPPS has shifted towards a biopsychosocial approach. While the study of psychotherapy for psychosomatic pain disorders has not been well studied, psychodynamic psychotherapy may help reduce the impact of the symptoms and improve social/occupational functioning ${ }^{44}$. The therapy helps increase the patient's awareness of maladaptive self-harming behaviors while steering them away from catastrophic thinking, leading to pain relief ${ }^{11}$ (Level of evidence 4, Grade C).

Cognitive behavioral therapy (CBT) has shown promise in the setting of chronic prostatitis/chronic pelvic pain syndrome (CP/CPPS) ${ }^{45}$. The use of CBT may also help patients challenge pain-distorted thinking, reduce avoidance of activities based on irrational fear of injury, and may potentially increase activity and reduce pain-related limitations ${ }^{11}$ (Level of evidence 4, Grade D).

Summary and recommendations: Lifestyle changes and physical therapies should be first-line therapy in all patients due to the non-invasive nature of treatment. Lifestyle changes include modification of aggravating activities, scrotal support and heat or cold therapies (Expert opinion). Physical therapy and acupuncture may improve CSP related to pelvic floor muscle dysfunction or referred pain from radiculopathies (Level of evidence 4, Grade $\mathrm{C}$ ). Psychological counseling may help treat maladaptive self-harming behaviors, prevent catastrophic thinking (Level of evidence 4, Grade $C$ ) and potentially decrease painrelated physical limitations (Level of evidence 4, Grade D).

\section{Medical management}

NSAIDs (4 weeks) (Grade 4C)

There is little specific evidence for the use of NSAIDS in the management of CSP and most of the data arises from general chronic pain literature. However, NSAIDs have anti-inflammatory effects, which may decrease nociceptive pain if there is a component of ongoing inflammation. Failing conservative strategies, a trial of 4 weeks of NSAIDs is a reasonable first line medical therapy (Level of evidence 4, Grade C).

Antibiotics (4 weeks) (Grade 3C)

While it is common sense that antibiotics should be offered to patients with culture proven infectious etiologies of CSP from their initial evaluation, antibiotics are commonly prescribed as empiric therapy for CSP as well ${ }^{1}$. However, the evidence behind this practice is quite limited. In 
one study assessing 55 patients presenting with CSP, a detailed infection screen was performed on each patient, including urine and semen cultures and screening for STIs ${ }^{46}$. Only 12 of the 55 (22\%) of patients presented with a significant bacterial colony count considered clinically relevant, while up to $64 \%$ of patients had previously received a course of antibiotics, suggesting a significant number of patients may have been over-treated (Level of evidence 3, Grade C).

However, in another study, 44 consecutive patients with idiopathic mild to moderate CSP and localized epididymal tenderness were treated with oral antibiotics (cephalosporins or quinolones as a first choice) as well as cessation of strenuous activity for 4 weeks ${ }^{47}$ (Level of evidence 3, Grade C). After this treatment course, all patients were fully recovered from scrotal pain and epididymal tenderness. While this is a small study, a 4-week course of empiric antibiotics is reasonable, especially in patients with tenderness localizable to the epididymis.

Patients with suspected infectious epididymitis should be treated empirically as per the Centers for Disease Control's guidelines, with coverage of Neisseria gonorrhea and Chlamydia trachomatis in men younger than 35, and coverage for coliform bacteria in those older than $35^{48}$ (Level of evidence 4, Grade C).

Neuropathic medications (4 weeks) (Grade 3C)

In patients with identified neuropathic pain, the recommended first-line treatment, as per the Canadian Pain Society consensus statement, consists of anticonvulsants and certain antidepressants ${ }^{49}$.

Gabapentin and pregabalin are gabapentinoids that belong to the anticonvulsant class of medications. They have been studied in large clinical trials largely in the setting of diabetic neuropathy and postherpetic neuralgia ${ }^{49}$. Both gabapentin and pregabalin bind presynaptic voltage-gated calcium channels in the dorsal horn of the spinal cord and are thought to interfere with pain transmission. In one small retrospective study comparing the efficacy of gabapentin and pregabalin in patients with chronic pelvic pain syndrome, gabapentin was found to be significantly more effective than pregabalin at controlling pain ${ }^{50}$. More than $75 \%$ of patients on gabapentin alone reported $\geq 50 \%$ improvement in symptoms versus only $40 \%$ on pregabalin alone, suggesting that gabapentin may be more effective than pregabalin in CPPS (Level of evidence 3, Grade C).

In another small retrospective series of 26 patients with idiopathic CSP, patients who had failed conservative therapies and failed anti-inflammatories and empiric antibiotics were prescribed either gabapentin or nortriptyline ${ }^{51}$. Gabapentin was started at $300 \mathrm{mg}$ daily and titrated by increasing the dose by $300 \mathrm{mg} /$ day up to a maximum of $1800 \mathrm{mg} /$ day depending on the clinical response and the side effects. Nortriptyline was started at $10 \mathrm{mg} /$ day then titrated up to a maximum of $150 \mathrm{mg} /$ day if required. Out of the patients on gabapentin, $61.5 \%$ of patients reported $\mathrm{a} \geq 50 \%$ improvement in pain, whereas $67 \%$ of patients on nortriptyline reported a $\geq 50 \%$ improvement in pain, suggesting that these medications may be effective in the treatment of CSP, though larger and ideally randomized controlled trials are needed (Level of evidence 3, Grade C).

Nortriptyline inhibits the reuptake of both noradrenalin and serotonin, but the exact mechanism in the setting of chronic pain is unknown. It is hypothesized that the antidepressant 
effects of nortriptyline may help treat any psychosomatic components of the CSP. However, if a significant psychiatric response to pain is present or if the pain presents with concomitant depression or anxiety, psychiatric referral is recommended. As with other urologic pain conditions such as IC/BPS, titrating from $10 \mathrm{mg} / \mathrm{day}$ to $50 \mathrm{mg} / \mathrm{day}$ has shown a reasonable compromise between efficacy for treating pain and side effects (Level of evidence 4, Grade C).

Nerve blockade (diagnostic and therapeutic) (Grade 4C) In patients who fail conservative and medical management, a spermatic cord blockade can be performed as a therapeutic measure. Yamamoto et al. reported on 3 patients with partial pain relief with spermatic cord blockade alone. They underwent further cord blockade 3 or more times and were quite satisfied with local block as a treatment alone ${ }^{36}$ (Level of evidence 4, Grade C).

The finding that nerve blocks provided temporary relief of CSP coupled with the effectiveness of MDSC prompted the further investigation of minimally invasive methods of longer-term cord blockade. In one pilot non-randomized study, 18 patients with CSP had a cord block with the injection of 100 units of OnabotulinumtoxinA (Botox) ${ }^{52}$ (Level of evidence 4, Grade $C$ ). Close to $72 \%$ of the men had pain relief lasting one month, $50 \%$ reported pain relief at 3 months and none had any ongoing relief of pain by 6 months. This is a potentially minimally invasive technique to provide a longer-term relief for the pain, but larger studies are needed to confirm these findings and to establish the role of Botox in the management of CSP.

Another promising minimally invasive treatment involves pulsed radiofrequency (pRF) denervation. Cohen and Foster first described the use of pRF directed to the genitofemoral, ilioinguinal, and iliohypogastric nerves in 3 patients with groin and testicular pain ${ }^{53}$ (Level of evidence 4, Grade C). They found that these patients reported complete pain relief at their 6month follow-up. Misra et al. further performed pRF of the spermatic cord in 9 patients ${ }^{54}$ (Level of evidence 4, Grade C). They found that 4 patients had complete pain resolution and that 1 had partial pain relief. Out of the patients that responded to the treatment, they continued to respond at a mean long-term follow-up of 9.6 months. Out of these two, small series, no side effects or procedure-related complications were noted. The use of pRF as a minimally invasive modality for CSP is certainly encouraging, however larger studies are needed before any evidence-based recommendations can be made.

Summary and recommendations: Second-line treatment of CSP should include a 4week trial of empiric antibiotics if infectious epididymitis is suspected (Level of evidence 3, Grade C), with or without NSAIDs (Level of evidence 4, Grade C). In patients with identified neuropathic pain, a 4-week trial of gabapentin or nortriptyline is recommended. The lowest recommended dose should be initially prescribed, with subsequent dose increases titrated to clinical benefit, while monitoring for adverse events (Expert opinion). If the initial selected medication (ie. gabapentin) is not effective, then an alternative medication (ie. Nortriptyline) should be considered (Expert opinion). Nerve blockade as a therapeutic 
measure should be considered prior to any surgical management as it may predict intervention success (Level of evidence 3, Grade C). Longer term nerve blockade modalities are still considered experimental, but early results are promising (Level of evidence 4, Grade D).

\section{Surgical management}

When conservative and medical management fail, surgery may be considered as the next treatment option. Surgical management of patients with CSP should if possible be directed at relieving the underlying causes for pain identified through the diagnostic evaluation.

Microsurgical vasovasostomy for post-vasectomy pain syndrome (PVPS) (Grade 3C) Persistent scrotal pain after vasectomy is a fairly rare complication. The American Urological Association (AUA) guideline on vasectomy states that 1-2\% of men undergoing vasectomy will develop $\mathrm{CSP}^{55}$. Other studies have reported up to $15 \%$ of men reporting new scrotal pain up to 7 months after the vasectomy ${ }^{56}$. The etiology of post vasectomy pain is still poorly understood. Potential theories include epididymal congestion, perineural fibrosis from scar tissue, inflammation from the leakage of antigenic vasal fluid, or vascular stasis ${ }^{57}$.

The published evidence on the management of post-vasectomy pain syndrome (PVPS) is still limited, with no level 1 data guiding management. However, as with the rest of CSP, it is reasonable to attempt conservative therapies and pharmacological treatment first before any surgical management proposed.

The literature on vasovasostomy (VV) or vasectomy reversal for PVPS comprises of small single-center studies. The concept of VV for PVPS seems to be intuitive. Through reestablishing continuity of the reproductive tract, this procedure aims to relieve epididymal obstruction as well as decrease the leakage of inflammatory vasal fluid from the testicular end. All studies on VV for PVPS have shown that nearly $100 \%$ of patients will have improvement in pain scores, with complete resolution ranging from $50-100 \%{ }^{58-61}$. In these studies, all patients underwent microsurgical vasovasostomies. 
Epididymectomy for PVPS and symptomatic epididymal cysts (Grade 3C)

Epididymectomy is another treatment for CSP that have been assessed in multiple small series in the literature. The success rates for epididymectomy in the setting of PVPS vary in the literature, ranging from $10 \%$ to $>90 \%{ }^{12,62-64}$. However, the success rates for CSP as a whole are less promising. Hori et al. evaluated 72 patients undergoing epididymectomy for CSP and compared patients with PVPS versus non-vasectomy patients ${ }^{65}$. In this study, 93\% of patients with PVPS had less or no pain post-epididymectomy, with an overall satisfaction rate of 93\%, compared to $75 \%$ and $62.5 \%$ respectively in the nonvasectomy group. Padmore et al. studied 57 patients after epididymectomy. They found a much higher cure rate in epididymectomy performed for symptomatic epididymal cysts (76\%) versus epididymitis (24\%). 22\% of the patients with epididymitis in this study further underwent orchiectomy for pain ${ }^{66}$. Davis et al. found that 9 out of 10 patients treated with epididymectomy for CSP required subsequent orchiectomy as a definitive treatment ${ }^{12}$. While the current published success rates of epididmyectomy specifically for PVPS do appear promising as well as in selected patients with palpable epididymal pathology (such as a painful cyst), it must be made clear to the patient that this procedure will make reconstruction of the reproductive tract impossible, possibly impacting future fertility.

Varicocele repair for symptomatic varicoceles (Grade 3C)

Varicoceles are a common finding, with a reported prevalence of $15 \%$ in the general population ${ }^{67}$. Though varicoceles are asymptomatic in many men, an estimated $10 \%$ of men with varicoceles will have $\mathrm{CSP}^{68}$. Due to the prevalence of varicoceles in the general population, it is important to assess and rule out any other causes of scrotal pain. Pain pathogenesis from varicoceles is poorly understood, with multiple theories including the dilatation of the pampiniform plexus causing compression of neural fibers, tissue ischemia secondary to venous stasis, increased scrotal temperature and oxidative stress in the testicular parenchyma ${ }^{69,70}$.

In patients who have failed conservative and medical management, varicocele repair, regardless of approach, has been shown in numerous small series to be effective, with reported improvement or complete resolution rates ranging from $80-100 \%{ }^{68,69,71}$. Though there are numerous approaches to varicocele repair, including inguinal, subinguinal, retroperitoneal, embolization and laparoscopic, these approaches have not been compared. We recommend that if varicocele repair is to be undertaken, a standard varicocelectomy should be performed using the accepted technique to surgically treat varicoceles for men with infertility.

Microsurgical denervation of the spermatic cord (MDSC) (Grade 3C)

MDSC was first reported by Devine and Schellhammer in 1978 as a means to treat testicular pain of unknown etiology ${ }^{5}$. In their initial report comprising of only 2 patients, they reported a 100\% complete resolution of pain in these patients. This surgical procedure has increased in popularity over the past decade with better understanding of the pathophysiology of CSP. Parakattil et al. identified abnormal Wallerian degeneration in the trifecta nerve complex of the spermatic cord, suggesting a neuroanatomical basis for the pain ${ }^{72}$. The purpose of MDSC is to transect the ilioinguinal nerve and all the nerves of the spermatic cord while preserving the testicular artery and the lymphatics, thus ablating the afferent neural pathways that may contribute to $\mathrm{CSP}^{23}$. 
Multiple retrospective studies have since been published looking at MDSC for CSP. The success rates for this procedure range from $71 \%$ to $96 \%$ 4,5,14,23,37,72. Benson et al. performed a retrospective review of 74 patients who underwent MDSC. They found that a positive response to spermatic cord blockade ( $\geq 50 \%$ reduction in pain) predicted for pain resolution in $75 \%$ of patients, suggesting that preoperative spermatic cord block response can predict for MDSC success ${ }^{35}$. The risks of the procedure, including persistent pain, persistent numbness, infection, bleeding, testicular atrophy, infertility, and hydrocele formation need to be discussed with the patient as part of the informed consent. Given the significant potential complications of this procedure, it should only be performed in dedicated centres with expertise in the MDSC technique.

Inguinal orchiectomy (Grade 3C)

Orchiectomy remains a surgical option and is usually considered as a last resort if other surgical approaches fail and the patient still has significant ongoing pain. The reported success rates of orchiectomy have been variable, ranging from $20 \%$ to $75 \%{ }^{12,25,36}$. If orchiectomy for CSP is to be performed, an inguinal approach should be used, as this has shown superior success rates as compared to the scrotal approach ${ }^{12}$. The higher success rate with the inguinal approach may reflect the ability to address any neuropathic components of pain related to the ilioinguinal nerve or the genital branch of the genitofemoral nerve ${ }^{40}$.

As this is an extirpative surgery, aside from the usual risks associated with surgery, specific risks of the procedure include increased risk of hypogonadism and infertility and this should be very carefully discussed with the patient and documented. Patients may have decreased satisfaction with the appearance of their genitalia after the procedure, and must also be counseled that CSP may persist despite orchiectomy ${ }^{40}$.

Summary and recommendations: Surgery should be considered after a trial of conservative therapies and pharmacological therapies have been attempted (Expert opinion). The choice of initial surgical approach should be directed by the likely etiology of pain. In patients with PVPS, multiple series have shown complete pain resolution rates ranging from $50-100 \%$ with microsurgical vasectomy reversal, and lower rates with epididymectomy with a range from 10-90\% (Level of evidence 3, Grade C). Though up to $15 \%$ of the male population will have a varicocele, only an estimated $10 \%$ will have associated CSP. In select patients with CSP associated with varicocele, varicocele repair has demonstrated success rates ranging from 80-100\% (Level of evidence 3, Grade C). MDSC has shown promise for idiopathic CSP as well as PVPS, with success rates ranging from 71-95\% (Level of evidence 3, Grade C), this procedure should only be performed in dedicated centres with expertise in the MDSC technique (Expert Opinion). A diagnostic spermatic cord block is recommended prior to MDSC as this may predict pain resolution success (Level of evidence 3, Grade C). Orchiectomy remains a surgical option in patients with pain refractory to all other interventions and should only be performed with an inguinal approach (Level of evidence 4, Grade $C$ ). Published success rates of inguinal orchiectomy range from $20-75 \%$ (Level of evidence 4 , Grade $C$ ). 


\section{Future directions and recommendations for best practice}

CSP is a debilitating and distressing condition that significantly impacts the quality of life of patients. It is also a common yet challenging condition to treat by urologists, with variable responses to current treatment. Emerging research show that lifestyle modification and the early introduction of medications such as tricyclic antidepressants and gabapentinoids may reduce the reliance on narcotic medications and empower patients with new coping strategies. New perspectives in chronic pain management have shifted from a disease model to a biopsychosocial model, emphasizing the importance of multidisciplinary care for this patient population, including referral for psychological and pain clinic assessments.

As further research on CSP continues, new treatments for CSP will continue to emerge. New therapies have shown promise, including minimally invasive nerve blockade strategies. Large, multi-center, controlled trials are necessary in this field to evaluate the efficacy of current and novel treatment approaches.

Evaluation and management of the patient with CSP should follow the algorithm highlighted in this guideline. Surgery in general, should be considered if conservative and medical approaches have been exhausted. MDSC appears to be the most promising surgical intervention currently for patients with a positive response to spermatic cord block and will spare the testicle, allowing for preservation of hormonal function.

\section{Conclusion}

Chronic scrotal pain is a common, complex, yet poorly understood condition that can be difficult to manage, as the etiology of pain is oftentimes unknown. A thorough diagnostic evaluation guided by a history and physical examination may help identify helpful adjunct tests that may clarify potential causes of pain. Lifestyle modifications, physical therapy, and psychotherapy may empower patients and help provide tools for better coping with this challenging condition. Non-narcotic pharmacological options have also been studied and show promise, especially in the setting of neuropathic pain. Even if these measures fail, there are now a number of promising surgical interventions available to urologists for CSP.

With this CUA best practices statement (BPS), a systematic approach to the evaluation and management of the patient with CSP is presented, through the best available evidence to date on the management of this challenging condition. 


\section{References}

1. Aljumaily A, Al-Khazraji H, Gordon A et al. Characteristics and Etiologies of Chronic Scrotal Pain: A Common but Poorly Understood Condition. Pain Res Manag 2017; 2017: 3829168.

2. Strebel RT, Leippold T, Luginbuehl T et al. Chronic scrotal pain syndrome: management among urologists in Switzerland. Eur Urol 2005; 47: 812-6.

3. Ciftci H, Savas M, Yeni E et al. Chronic orchialgia and associated diseases. Current Urology 2010; 4: 67-70.

4. Heidenreich A, Olbert P, Engelmann UH. Management of chronic testalgia by microsurgical testicular denervation. Eur Urol 2002; 41: 392-7.

5. Devine CJ, Jr., Schellhammer PF. The use of microsurgical denervation of the spermatic cord for orchialgia. Trans Am Assoc Genitourin Surg 1978; 70: 149-51.

6. Levine LA, Hoeh MP. Evaluation and management of chronic scrotal content pain. Curr Urol Rep 2015; 16: 36.

7. Fall M, Baranowski AP, Elneil S et al. EAU guidelines on chronic pelvic pain. Eur Urol 2010; 57: 35-48.

8. Aljumaily A, Wu C, Al-Khazraji H et al. Quality of life in men with chronic scrotal pain. Canadian Journal of Pain 2017; 1: 106-11.

9. Schover LR. Psychological factors in men with genital pain. Cleve Clin J Med 1990; 57: 697-700.

10. Turk DC, Okifuji A. Perception of traumatic onset, compensation status, and physical findings: impact on pain severity, emotional distress, and disability in chronic pain patients. $J$ Behav Med 1996; 19: 435-53.

11. Lian F, Shah A, Mueller B et al. Psychological perspectives in the patient with chronic orchialgia. Transl Androl Urol 2017; 6: S14-S9.

12. Davis BE, Noble MJ, Weigel JW et al. Analysis and management of chronic testicular pain. J Urol 1990; 143: 936-9.

13. Levine L. Chronic orchialgia: evaluation and discussion of treatment options. Ther Adv Urol 2010; 2: 209-14.

14. Levine LA, Matkov TG. Microsurgical denervation of the spermatic cord as primary surgical treatment of chronic orchialgia. J Urol 2001; 165: 1927-9.

15. Rottenstreich M, Glick Y, Gofrit ON. Chronic scrotal pain in young adults. BMC Res Notes 2017; 10: 241.

16. Nickel JC, Teichman JM, Gregoire $\mathrm{M}$ et al. Prevalence, diagnosis, characterization, and treatment of prostatitis, interstitial cystitis, and epididymitis in outpatient urological practice: the Canadian PIE Study. Urology 2005; 66: 935-40.

17. Treede RD, Jensen TS, Campbell JN et al. Neuropathic pain: redefinition and a grading system for clinical and research purposes. Neurology 2008; 70: 1630-5.

18. Masarani M, Cox R. The aetiology, pathophysiology and management of chronic orchialgia. BJU Int 2003; 91: 435-7. 
19. Kehlet H, Werner MU. [Role of paracetamol in the acute pain management]. Drugs 2003; 63 Spec No 2: 15-22.

20. Sigalos JT, Pastuszak AW. Chronic orchialgia: epidemiology, diagnosis and evaluation. Transl Androl Urol 2017; 6: S37-S43.

21. Tatem A, Kovac JR. Chronic scrotal pain and microsurgical spermatic cord denervation: tricks of the trade. Transl Androl Urol 2017; 6: S30-S6.

22. Wagenlehner FM, van Till JW, Magri V et al. National Institutes of Health Chronic Prostatitis Symptom Index (NIH-CPSI) symptom evaluation in multinational cohorts of patients with chronic prostatitis/chronic pelvic pain syndrome. Eur Urol 2013; 63: 953-9.

23. Strom KH, Levine LA. Microsurgical denervation of the spermatic cord for chronic orchialgia: long-term results from a single center. The Journal of urology 2008; 180: 949-53. 24. Schneiderman H, Voytovich A. (Self-) palpation orchitis. J Gen Intern Med 1988; 3: 97. 25. Costabile RA, Hahn M, McLeod DG. Chronic orchialgia in the pain prone patient: the clinical perspective. J Urol 1991; 146: 1571-4.

26. Krsmanovic A, Tripp DA, Nickel JC et al. Psychosocial mechanisms of the pain and quality of life relationship for chronic prostatitis/chronic pelvic pain syndrome (CP/CPPS). Can Urol Assoc J 2014; 8: 403-8.

27. Hu JC, Link CL, McNaughton-Collins $M$ et al. The association of abuse and symptoms suggestive of chronic prostatitis/chronic pelvic pain syndrome: results from the Boston Area Community Health survey. J Gen Intern Med 2007; 22: 1532-7.

28. Nickel JC, Siemens DR, Nickel KR et al. The patient with chronic epididymitis: characterization of an enigmatic syndrome. J Urol 2002; 167: 1701-4.

29. Nickel JC. Chronic epididymitis: a practical approach to understanding and managing a difficult urologic enigma. Rev Urol 2003; 5: 209-15.

30. Nickel JC. The Pre and Post Massage Test (PPMT): a simple screen for prostatitis. Tech Urol 1997; 3: 38-43.

31. Kromann-Andersen B, Hansen LB, Larsen PN et al. Clinical versus ultrasonographic evaluation of scrotal disorders. Br J Urol 1988; 61: 350-3.

32. van Haarst EP, van Andel G, Rijcken TH et al. Value of diagnostic ultrasound in patients with chronic scrotal pain and normal findings on clinical examination. Urology 1999; 54: 106872.

33. London NJ, Smart JG, Kinder RB et al. Prospective study of routine scrotal ultrasonography in urological practice. Br J Urol 1989; 63: 416-9.

34. Cho SH, Min SK, Lee ST. Associations of ultrasonographic features with scrotal pain after vasectomy. Korean J Urol 2011; 52: 782-6.

35. Benson JS, Abern MR, Larsen S et al. Does a positive response to spermatic cord block predict response to microdenervation of the spermatic cord for chronic scrotal content pain? $J$ Sex Med 2013; 10: 876-82.

36. Yamamoto M, Hibi H, Katsuno S et al. Management of chronic orchialgia of unknown etiology. International journal of urology : official journal of the Japanese Urological Association 1995; 2: 47-9. 
37. Larsen SM, Benson JS, Levine LA. Microdenervation of the spermatic cord for chronic scrotal content pain: single institution review analyzing success rate after prior attempts at surgical correction. J Urol 2013; 189: 554-8.

38. Rusz A, Pilatz A, Wagenlehner F et al. Influence of urogenital infections and inflammation on semen quality and male fertility. World J Urol 2012; 30: 23-30.

39. Michel V, Duan Y, Stoschek E et al. Uropathogenic Escherichia coli causes fibrotic remodelling of the epididymis. J Pathol 2016; 240: 15-24.

40. Lowe G. Extirpative surgery for chronic orchialgia: is there a role? Transl Androl Urol 2017; 6: S2-S5.

41. Fitzgerald MP, Anderson RU, Potts J et al. Randomized multicenter feasibility trial of myofascial physical therapy for the treatment of urological chronic pelvic pain syndromes. $J$ Urol 2013; 189: S75-85.

42. Doubleday KL, Kulig K, Landel R. Treatment of testicular pain using conservative management of the thoracolumbar spine: a case report. Arch Phys Med Rehabil 2003; 84: 1903-5. 43. Chen R, Nickel JC. Acupuncture ameliorates symptoms in men with chronic prostatitis/chronic pelvic pain syndrome. Urology 2003; 61: 1156-9; discussion 9.

44. Abbass A, Kisely S, Kroenke K. Short-term psychodynamic psychotherapy for somatic disorders. Systematic review and meta-analysis of clinical trials. Psychother Psychosom 2009; 78: $265-74$.

45. Nickel JC, Mullins C, Tripp DA. Development of an evidence-based cognitive behavioral treatment program for men with chronic prostatitis/chronic pelvic pain syndrome. World $J$ Urol 2008; 26: 167-72.

46. Strebel RT, Schmidt C, Beatrice J et al. Chronic scrotal pain syndrome (CSPS): the widespread use of antibiotics is not justified. Andrology 2013; 1: 155-9.

47. Lai Y, Yu Z, Shi B et al. Chronic scrotal pain caused by Mild Epididymitis:Report of a series of 44 cases. Pak J Med Sci 2014; 30: 638-41.

48. Taylor SN. Epididymitis. Clin Infect Dis 2015; 61 Suppl 8: S770-3.

49. Moulin D, Boulanger A, Clark AJ et al. Pharmacological management of chronic neuropathic pain: revised consensus statement from the Canadian Pain Society. Pain Res Manag 2014; 19: 328-35.

50. Agarwal MM, Elsi Sy M. Gabapentenoids in pain management in urological chronic pelvic pain syndrome: Gabapentin or pregabalin? Neurourol Urodyn 2017; 36: 2028-33.

51. Sinclair AM, Miller B, Lee LK. Chronic orchialgia: consider gabapentin or nortriptyline before considering surgery. Int J Urol 2007; 14: 622-5.

52. Khambati A, Lau S, Gordon A et al. OnabotulinumtoxinA (Botox) nerve blocks provide durable pain relief for men with chronic scrotal pain: a pilot open-label trial. J Sex Med 2014; 11: 3072-7.

53. Cohen SP, Foster A. Pulsed radiofrequency as a treatment for groin pain and orchialgia. Urology 2003; 61: 645.

54. Misra S, Ward S, Coker C. Pulsed radiofrequency for chronic testicular pain-a preliminary report. Pain Med 2009; 10: 673-8. 
55. Sharlip ID, Belker AM, Honig S et al. Vasectomy: AUA guideline. J Urol 2012; 188: 2482-91.

56. Leslie TA, Illing RO, Cranston DW et al. The incidence of chronic scrotal pain after vasectomy: a prospective audit. BJU Int 2007; 100: 1330-3.

57. Tan WP, Levine LA. An overview of the management of post-vasectomy pain syndrome. Asian J Androl 2016; 18: 332-7.

58. Polackwich AS, Tadros NN, Ostrowski KA et al. Vasectomy Reversal for Postvasectomy Pain Syndrome: A Study and Literature Review. Urology 2015; 86: 269-72.

59. Horovitz D, Tjong V, Domes T et al. Vasectomy reversal provides long-term pain relief for men with the post-vasectomy pain syndrome. The Journal of urology 2012; 187: 613-7.

60. Nangia AK, Myles JL, Thomas AJ. Vasectomy reversal for the post-vasectomy pain syndrome: a clinical and histological evaluation. J Urol 2000; 164: 1939-42.

61. Myers SA, Mershon CE, Fuchs EF. Vasectomy reversal for treatment of the postvasectomy pain syndrome. J Urol 1997; 157: 518-20.

62. Chen TF, Ball RY. Epididymectomy for post-vasectomy pain: histological review. Br J Urol 1991; 68: 407-13.

63. West AF, Leung HY, Powell PH. Epididymectomy is an effective treatment for scrotal pain after vasectomy. BJU Int 2000; 85: 1097-9.

64. Calleary JG, Masood J, Hill JT. Chronic epididymitis: is epididymectomy a valid surgical treatment? Int J Androl 2009; 32: 468-72.

65. Hori S, Sengupta A, Shukla CJ et al. Long-term outcome of epididymectomy for the management of chronic epididymal pain. J Urol 2009; 182: 1407-12.

66. Padmore DE, Norman RW, Millard OH. Analyses of indications for and outcomes of epididymectomy. J Urol 1996; 156: 95-6.

67. Thomason AM, Fariss BL. The prevalence of varicoceles in a group of healthy young men. Mil Med 1979; 144: 181-2.

68. Peterson AC, Lance RS, Ruiz HE. Outcomes of varicocele ligation done for pain. $J$ Urol 1998; 159: 1565-7.

69. Owen RC, McCormick BJ, Figler BD et al. A review of varicocele repair for pain. Transl Androl Urol 2017; 6: S20-S9.

70. Chen LK, Chen SS. Risk factors for developing pain in normospermic patients with varicocoele. Int J Androl 2012; 35: 176-80.

71. Elzanaty S, Johansen CE. Microsurgical Varicocele Repair on Men with Grade III Lesions and Chronic Dull Scrotal Pain: A Pilot Study. Curr Urol 2015; 8: 29-31.

72. Parekattil SJ, Gudeloglu A, Brahmbhatt JV et al. Trifecta nerve complex: potential anatomical basis for microsurgical denervation of the spermatic cord for chronic orchialgia. The Journal of urology 2013; 190: 265-70.

73. Harirforoosh S, Asghar W, Jamali F. Adverse effects of nonsteroidal antiinflammatory drugs: an update of gastrointestinal, cardiovascular and renal complications. J Pharm Pharm Sci 2013; 16: 821-47. 
74. $\quad$ Fish DN. Fluoroquinolone adverse effects and drug interactions. Pharmacotherapy 2001; 21: 253S-72S.

75. Moskovitz BL. Clinical adverse effects during ceftriaxone therapy. Am J Med 1984; 77: 84-8.

76. Lau CY, Qureshi AK. Azithromycin versus doxycycline for genital chlamydial infections: a meta-analysis of randomized clinical trials. Sex Transm Dis 2002; 29: 497-502.

77. Backonja M, Glanzman RL. Gabapentin dosing for neuropathic pain: evidence from randomized, placebo-controlled clinical trials. Clin Ther 2003; 25: 81-104.

78. Waldfogel JM, Nesbit SA, Dy SM et al. Pharmacotherapy for diabetic peripheral neuropathy pain and quality of life: A systematic review. Neurology 2017; 88: 1958-67. 


\section{Figures and Tables}

Fig. 1. Diagnostic evaluation algorithm. CESI: chronic epididymitis symptom index; CPPS: chronic pelvic pain syndrome; US: ultrasound.

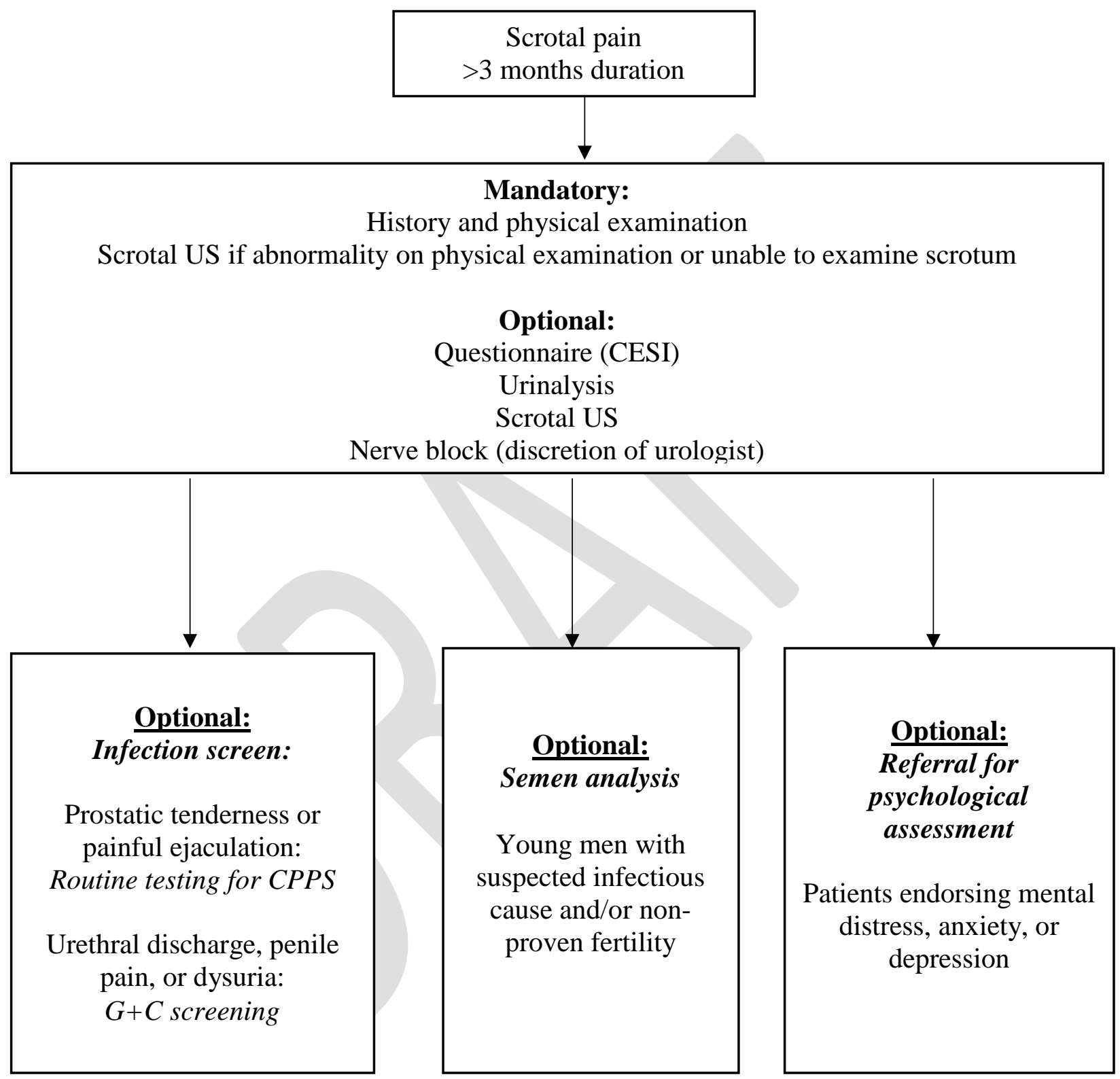


Fig. 2. Conservative and medical management of idiopathic chronic scrotal pain.

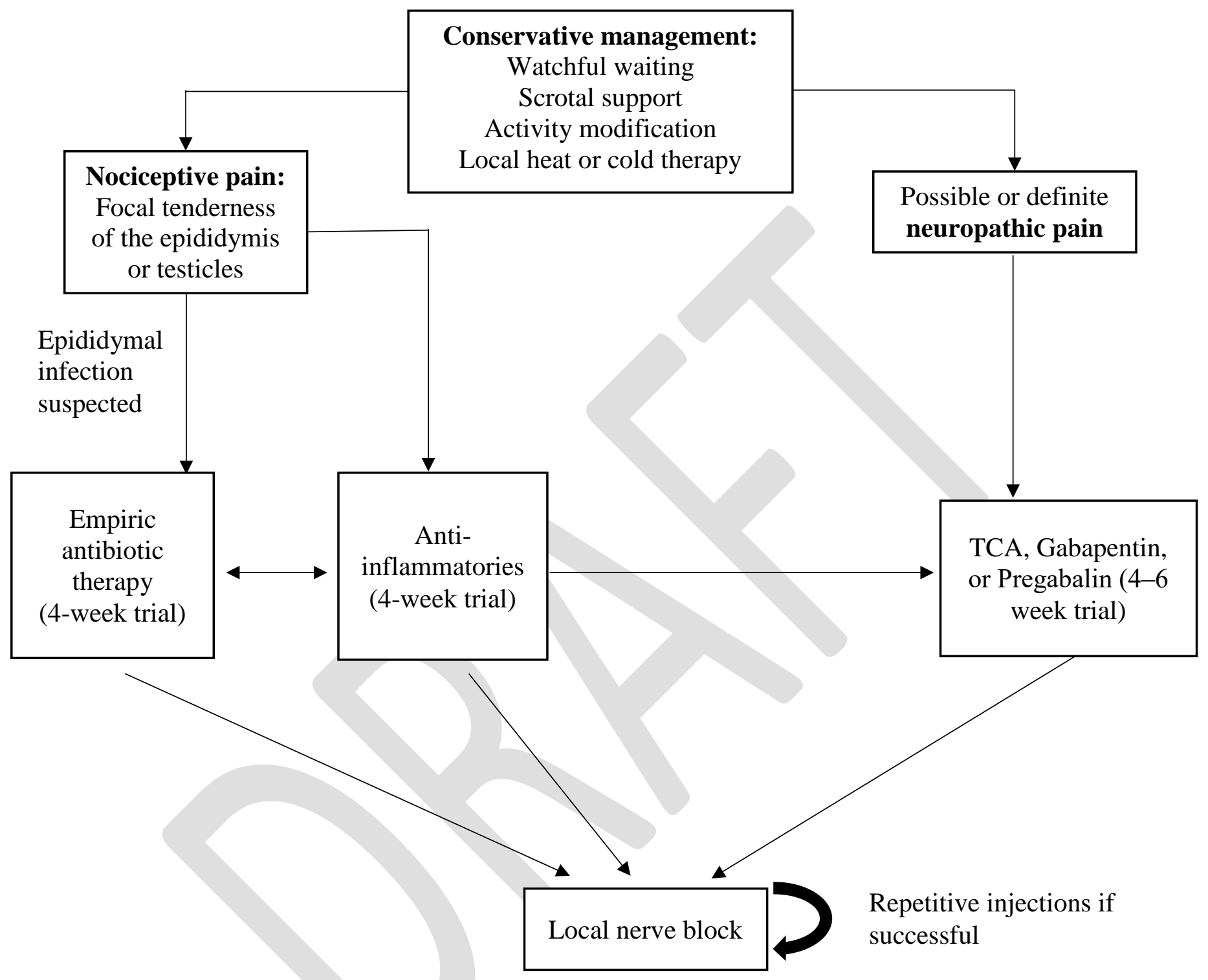




\begin{tabular}{|c|c|c|c|}
\hline Medication class & Dosage & Reported efficacy & Common side effects \\
\hline $\begin{array}{l}\text { Non-steroidal anti- } \\
\text { inflammatory } \\
\text { drugs (NSAIDs) }\end{array}$ & $\begin{array}{l}\text { Ibuprofen } 400- \\
600 \text { mg po q6h } \\
\text { Naproxen } 500 \\
\text { mg po BID }\end{array}$ & Unknown & $\begin{array}{l}\text { Dyspepsia, gastro- } \\
\text { duodenal ulcers, acute } \\
\text { and chronic renal } \\
\text { failure }(1-5 \%)^{73}\end{array}$ \\
\hline Antibiotics & $\begin{array}{l}\text { Levofloxacin } \\
500 \text { mg po daily } \\
\text { x } 10 \text { days } \\
\text { If risk of } \\
\text { sexually } \\
\text { transmitted } \\
\text { infections: } \\
\text { Ceftriaxone } 250 \\
\text { mg IM + } \\
\text { doxycycline } 100 \\
\text { mg po BID x } 10 \\
\text { days }\end{array}$ & $26-100 \%{ }^{28,47}$ & $\begin{array}{l}\text { Levofloxacin: nausea } \\
\text { (4-8\%), diarrhea } \\
\text { (2\%), headache (1- } \\
\text { 2\%), dizziness, } \\
\text { elevated } \\
\text { transaminases (2- } \\
3 \%)^{74} \\
\text { Ceftriaxone: } \\
\text { Gastrointestinal } \\
\text { (3.5\%), } \\
\text { hypersensitivity(3\%) } \\
\text { Doxycycline: } \\
\text { Diarrhea, abdominal } \\
\text { pain, fatigue }\end{array}$ \\
\hline Gabapentinoids & $\begin{array}{l}\text { Gabapentin } 300 \\
\text { mg po daily, } \\
\text { uptitrate by } 300 \\
\text { mg/day up to } \\
\text { maximum of } \\
1800 \text { mg/day }\end{array}$ & $\begin{array}{l}61.5-75 \% \text { of patients with } \\
\geq 50 \% \text { improvement in } \\
\text { symptoms }\end{array}$ & $\begin{array}{l}\text { Sedation, dizziness, } \\
\text { nausea, } \\
\text { gastrointestinal } \\
\text { upset }^{77}\end{array}$ \\
\hline $\begin{array}{l}\text { Tricyclic } \\
\text { antidepressants }\end{array}$ & $\begin{array}{l}\text { Nortriptyline } 10 \\
\text { mg po TID, } \\
\text { uptitrate by } 10 \\
\text { mg daily to a } \\
\text { maximum of } \\
150 \text { mg/day }\end{array}$ & $\begin{array}{l}67 \% \text { of patients with } \geq 50 \% \\
\text { improvement in symptoms }\end{array}$ & $\begin{array}{l}\text { Sedation, dry mouth, } \\
\text { dizziness, insomnia }^{78}\end{array}$ \\
\hline
\end{tabular}




\begin{tabular}{|c|c|}
\hline Intervention & Success rate \\
\hline OnabotulinumtoxinA cord blockade & $\begin{array}{l}\geq 50 \% \text { with partial or complete resolution of pain at } 3 \\
\text { months followup }\end{array}$ \\
\hline Pulsed radiofrequency denervation & $\begin{array}{l}56-100 \% \text { partial or complete resolution of pain at 3-6 } \\
\text { months followup }\end{array}$ \\
\hline $\begin{array}{l}\text { Microsurgical vasovasostomy for } \\
\text { PVPS }\end{array}$ & $50-100 \%$ complete resolution of pain ${ }^{58-60}$ \\
\hline $\begin{array}{l}\text { Epididymectomy for PVPS and } \\
\text { symptomatic epididymal cysts }\end{array}$ & $\begin{array}{l}\text { 10-90\% with partial or complete resolution of } \\
\text { pain }^{12,62-64}\end{array}$ \\
\hline $\begin{array}{l}\text { Varicocele repair for symptomatic } \\
\text { varicoceles }\end{array}$ & $\begin{array}{l}80-100 \% \text { with partial or complete resolution of } \\
\text { pain }^{68,69,71}\end{array}$ \\
\hline $\begin{array}{l}\text { Microsurgical denervation of the } \\
\text { spermatic cord (MDSC) }\end{array}$ & $\begin{array}{l}\text { 71-96\% with partial or complete resolution of } \\
\text { pain }^{4,5,14,23,37,72}\end{array}$ \\
\hline Inguinal orchiectomy & $\begin{array}{l}20-75 \% \text { with partial or complete resolution of } \\
\text { pain }^{12,25,36}\end{array}$ \\
\hline
\end{tabular}

Table 3. Etiologies of chronic scrotal pain ${ }^{1}$

\begin{tabular}{|l|c|}
\hline Causes & Percentage of patients \\
\hline Vasectomy & $20.61 \%$ \\
\hline Trauma & $12.21 \%$ \\
\hline Infection & $11.45 \%$ \\
\hline Hernia repair & $4.58 \%$ \\
\hline Epididymal cyst & $1.52 \%$ \\
\hline $\begin{array}{l}\text { Other identified causes } \\
\text { (Hydrocelectomy, TURP, orchiectomy, donor nephrectomy) }\end{array}$ & $6.10 \%$ \\
\hline Unknown & $43.51 \%$ \\
\hline
\end{tabular}

TURP: transurethral resection of the prostate. 